\title{
Permutation transformations of tensors with an application
}

\author{
Yao-Tang Li ${ }^{*}{ }^{\dagger}$, Zheng-Bo Li ${ }^{\dagger}$, Qi-Long Liu ${ }^{\dagger}$ and Qiong Liu ${ }^{\dagger}$
}

${ }^{*}$ Correspondence: liyaotang@ynu.edu.cn

†Yao-Tang Li, Zheng-Bo Li,

Qi-Long Liu and Qiong Liu

contributed equally to this

work

School of Mathematics

and Statistics, Yunnan

University, Kunming 650091,

People's Republic of China

\begin{abstract}
The permutation transformation of tensors is introduced and its basic properties are discussed. The invariance under permutation transformations is studied for some important structure tensors such as symmetric tensors, positive definite (positive semidefinite) tensors, Z-tensors, $M$-tensors, Hankel tensors, $P$-tensors, $B$-tensors and $H$-tensors. Finally, as an application of permutation transformations of tensors, the canonical form theorem of tensors is given. The theorem shows that some problems of higher dimension tensors can be translated into the corresponding problems of lower dimension weakly irreducible tensors so as to handle easily.
\end{abstract}

Keywords: Permutation transformation, Structure tensor, Weakly irreducible tensor, Canonical form

Mathematics Subject Classification: 15A69, 12E05, 12E10

\section{Background}

The study of tensors with their various applications has attracted extensive attention and interest, since the work of Qi (2005) and Lim (2005). Lately, the research topic on structure tensors has also attracted much attention, such as symmetric tensors (Qi 2005), $P\left(P_{0}\right)$-tensors (Song and Qi 2014), B(B0)-tensors (Song and Qi 2014), Z-tensors (Zhang et al. 2014), (strong) $M$-tensors (Zhang et al. 2014), $H$-tensors (Li et al. 2014) and so on. In the researches on tensors with its application, the reducibility and higher dimension of tensors are two important factors to cause difficulties. Therefore, it is interesting that how to translate problems of higher dimension reducible tensors into the corresponding problems of lower dimension irreducible tensors.

As we all know, the permutation transformation of matrices plays a very important role in linear algebra and matrix theory. Some problems of higher dimension reducible matrices can be translated into the corresponding problems of lower dimension irreducible matrices by using the permutation transformation of matrices. Inspired by this, we introduce permutation transformations of tensors, and discuss its basic properties and and their applications in this paper.

In the next section, we will introduce the permutation transformation of tensors and give its expression. In third section, we will discuss basic properties of permutation transformations of tensors. In fourth section, we will discuss the invariance under

(c) The Author(s) 2016. This article is distributed under the terms of the Creative Commons Attribution 4.0 International License (http://creativecommons.org/licenses/by/4.0/), which permits unrestricted use, distribution, and reproduction in any medium, provided you give appropriate credit to the original author(s) and the source, provide a link to the Creative Commons license, and indicate if changes were made. 
permutation transformations for some important structure tensors such as symmetric tensors, positive definite tensors, $M$-tensors, Hankel tensors, $P$-tensors, $B$-tensors, $H$-tensors and so on. In fifth section, we will give the canonical form theorem of tensors and a numerical example which shows that some problems of higher dimension tensors can be translated into the corresponding problems of lower dimension weakly irreducible tensors by using permutation transformations. Finally, we draw some conclusions in the last section.

\section{Permutation transformations of tensors and its expression}

For a positive integer $n$, let $[n]=\{1,2, \ldots, n\}$. An order $m$ tensor $\mathcal{A}=\left(a_{i_{1} \ldots i_{m}}\right) \in$ $\mathbb{C}^{n_{1} \times n_{2} \times \cdots \times n_{m}}$ is a multidimensional array with $n_{1} n_{2} \ldots n_{m}$ entries, where $i_{j} \in\left[n_{j}\right], j \in[m]$. Especially, an order $m$ dimension $n$ tensor $\mathcal{A}=\left(a_{i_{1} \ldots i_{m}}\right)$ over the complex field $\mathbb{C}$ (real field $\mathbb{R}$ ) consists of $n^{m}$ complex (real) entries:

$$
a_{i_{1} \ldots i_{m}} \in \mathbb{C}(\mathbb{R})
$$

where $i_{j} \in[n]$ for $j \in[m]$ (Chang et al. 2008; De Lathauwer et al. 2000; Liu et al. 2010; Ng et al. 2009; Zhang and Golub 2001). It is obvious that a matrix is an order 2 tensor. We shall denote the set of all complex (real) order $m$ dimension $n$ tensors by $\mathbb{C}^{[m, n]}\left(\mathbb{R}^{[m, n]}\right.$, respectively).

Definition 1 Let $\mathcal{A}=\left(a_{i_{1} \ldots i_{m}}\right) \in \mathbb{C}^{[m, n]}, \mathcal{B}=\left(b_{i_{1} \ldots i_{m}}\right) \in \mathbb{C}^{[m, n]}$, and $k \in \mathbb{C}$. Define

(i) $\mathcal{A}+\mathcal{B}=\left(a_{i_{1} \ldots i_{m}}+b_{i_{1} \ldots i_{m}}\right)$.

(ii) $k \mathcal{A}=\left(k a_{i_{1} \ldots i_{m}}\right)$.

Remark 1 Obviously, both $\mathbb{C}^{[m, n]}$ and $\mathbb{R}^{[m, n]}$ are linear spaces about the addition and the multiplication in Definition 1.

Definition 2 (Qi 2005) A tensor $\mathcal{A}=\left(a_{i_{1} \ldots i_{m}}\right) \in \mathbb{R}^{[m, n]}$ is called a symmetric tensor if its entries $a_{i_{1} \ldots i_{m}}$ are invariant under any permutation of their indices.

Denote the set of all real order $m$ dimension $n$ symmetric tensors by $\mathbb{S}^{[m, n]}$. Furthermore, $\mathbb{S}^{[m, n]}$ is a linear subspace of $\mathbb{R}^{[m, n]}$. An order $m$ dimension $n$ tensor is called the unit tensor (Yang and Yang 2010), denoted by $\mathcal{I}$, if its entries are $\delta_{i_{1} \ldots i_{m}}$ for $i_{1}, \ldots, i_{m} \in[n]$, where

$$
\delta_{i_{1} \ldots i_{m}}= \begin{cases}1, & \text { if } i_{1}=\cdots=i_{m} \\ 0, & \text { otherwise. }\end{cases}
$$

Let $\mathcal{A}=\left(a_{i_{1} \ldots i_{m}}\right) \in \mathbb{R}^{[m, n]}$ and $x \in \mathbb{R}^{n}$. Then $\mathcal{A} x^{m}$ is a homogeneous polynomial of degree $m$, defined by

$$
\mathcal{A} x^{m}=\sum_{i_{1}, \ldots, i_{m} \in[n]} a_{i_{1} \ldots i_{m}} x_{i_{1}} \ldots x_{i_{m}} .
$$

A tensor $\mathcal{A} \in \mathbb{R}^{[m, n]}$ is called positive semidefinite (Song and Qi 2014) if for any vector $x \in \mathbb{R}^{n}, \mathcal{A} x^{m} \geq 0$, and it is called positive definite if for any nonzero vector $x \in \mathbb{R}^{n}, \mathcal{A} x^{m}>0$. 
Now, we give the definition of permutation transformation of tensors.

Definition 3 Let $\mathcal{A}=\left(a_{i_{1} \ldots i_{m}}\right) \in \mathbb{C}^{[m, n]}$ and $\pi$ be a permutation on [n], we define $P_{\pi}: \mathbb{C}^{[m, n]} \rightarrow \mathbb{C}^{[m, n]}$ by

$$
P_{\pi}(\mathcal{A})=\left(a_{\pi\left(i_{1}\right) \ldots \pi\left(i_{m}\right)}\right) .
$$

$P_{\pi}$ is called as a permutation transformation on $\mathbb{C}^{[m, n]}$, and is simply called as a permutation transformation. $P_{\pi}(\mathcal{A})$ is called as the image of $\mathcal{A}$ under $P_{\pi}$.

Remark $2 P_{\pi}$ is called as a permutation transformation on $\mathbb{R}^{[m, n]}$ if $\mathbb{C}^{[m, n]}$ is replaced by $\mathbb{R}^{[m, n]}$ in Definition 3.

Definition 4 Let $\mathcal{A}=\left(a_{i_{1} \ldots i_{m}}\right) \in \mathbb{C}^{[m, n]}$ and $\pi^{-1}$ be the inverse permutation of $\pi$ on $[n]$, we define $P_{\pi}^{-1}: \mathbb{C}^{[m, n]} \rightarrow \mathbb{C}^{[m, n]}$ by

$$
P_{\pi}^{-1}(\mathcal{A})=P_{\pi^{-1}}(\mathcal{A})
$$

$P_{\pi}^{-1}$ is called as the inverse permutation transformation of $P_{\pi}$ on $\mathbb{C}^{[m, n]}$, and is simply called as the inverse permutation transformation.

For further discussing property of the permutation transformation of tensors, we introduce the following general product of two $n$-dimensional tensors defined in Shao (2013). For the sake of simplicity, we sometime use the following "condensed notation" for the subscripts of the tensor. For example, we will write $a_{i_{1} i_{2} \ldots i_{m}}$ as $a_{i_{1} \alpha}$, where $\alpha=i_{2} \ldots i_{m} \in[n]^{m-1}$ and $[n]^{m-1}$ is $m-1$ dimensional array whose every element varies from 1 to $n$.

Definition 5 (Shao 2013) Let $\mathcal{A}$ and $\mathcal{B}$ be order $m \geq 2$ and order $k \geq 1$, dimension $n$ tensors, respectively. Define the product $\mathcal{A} \cdot \mathcal{B}$ (sometimes simplified as $\mathcal{A B}$ ) to be the following tensor $\mathcal{C}$ of order $(m-1)(k-1)+1$ and dimension $n$,

$$
c_{i \alpha_{1} \ldots \alpha_{m-1}}=\sum_{i_{2}, \ldots, i_{m} \in[n]} a_{i i_{2} \ldots i_{m}} b_{i_{2} \alpha_{1}} \ldots b_{i_{m} \alpha_{m-1}}
$$

where $i \in[n], \alpha_{1}, \ldots, \alpha_{m-1} \in[n]^{k-1}$.

Especially, when $P=\left(p_{i j}\right)$ and $Q=\left(q_{i j}\right)$ are both matrices, we have the following formula (Shao 2013):

$$
(P \mathcal{A Q})_{i_{1} \ldots i_{m}}=\sum_{j_{1}, \ldots, j_{m} \in[n]} a_{j_{1} \ldots j_{m}} p_{i_{1} j_{1}} q_{j_{2} i_{2}} \ldots q_{j_{m} i_{m}} .
$$

Definition 6 (Shao 2013) Let $\mathcal{A}, \mathcal{B} \in \mathbb{C}^{[m, n]}$. If there exists a permutation $\pi$ on the set $[n]$, and the corresponding permutation matrix $P=\left(P_{i j}\right)$ (where $P_{i j}=1 \Longleftrightarrow j=\pi(i)$; $P_{i j}=0$, otherwise.) such that $\mathcal{B}=P \mathcal{A} P^{T}$, then we say that $\mathcal{A}$ and $\mathcal{B}$ are permutational similar.

Remark 3 (Shao 2013) If $\mathcal{A}, \mathcal{B}$ are permutational similar, then 
(i) $b_{i_{1} \ldots i_{m}}=a_{\pi\left(i_{1}\right) \ldots \pi\left(i_{m}\right)}$

(ii) $P \mathcal{I} P^{T}=\mathcal{I}$.

Definition 7 (Shao 2013) Let $\mathcal{A}, \mathcal{B} \in \mathbb{C}^{[m, n]}$. Suppose that there exist two matrices $P$ and $Q$ of dimension $n$ with $P \mathcal{I} Q=\mathcal{I}$ such that $\mathcal{B}=P \mathcal{A} Q$, then we say that the two tensors $\mathcal{A}$ and $\mathcal{B}$ are similar.

Now, we present the relationship between the permutation transformation of tensors and permutational similar.

Theorem 1 Let $\mathcal{A}, \mathcal{B} \in \mathbb{C}^{[m, n]}$. Then $\mathcal{A}$ and $\mathcal{B}$ are permutational similar if and only if there exists a permutation $\pi$ on $[n]$ such that $\mathcal{B}=P_{\pi}(\mathcal{A})$.

Proof Assume that $\mathcal{A}$ and $\mathcal{B}$ are permutational similar. By Remark 3, there exists a permutation $\pi$ on $[n]$ such that $b_{i_{1} \ldots i_{m}}=a_{\pi\left(i_{1}\right) \ldots \pi\left(i_{m}\right)}$. Define a permutation transformation $P_{\pi}: \mathbb{C}^{[m, n]} \rightarrow \mathbb{C}^{[m, n]}$ by

$$
P_{\pi}(\mathcal{A})=\left(a_{\pi\left(i_{1}\right) \ldots \pi\left(i_{m}\right)}\right)
$$

which implies $\mathcal{B}=P_{\pi}(\mathcal{A})$.

On the other hand, assume that there exists a permutation transformation $P_{\pi}$ such that $\mathcal{B}=P_{\pi}(\mathcal{A})$, then

$$
b_{i_{1} \ldots i_{m}}=a_{\pi\left(i_{1}\right) \ldots \pi\left(i_{m}\right)} .
$$

Let permutation matrix $P=P_{\pi}\left(P_{i j}=1 \Longleftrightarrow j=\pi(i) ; P_{i j}=0\right.$, otherwise. $)$ corresponding to $\pi$. Then

$$
\begin{aligned}
\left(P \mathcal{A} P^{T}\right)_{i_{1} \ldots i_{m}} & =\sum_{j_{1}, \ldots, j_{m} \in[n]} a_{j_{1} \ldots j_{m}} P_{i_{1} j_{1}}\left(P^{T}\right)_{j_{2} i_{2}} \ldots\left(P^{T}\right)_{j_{m} i_{m}} \\
& =a_{\pi\left(i_{1}\right) \ldots \pi\left(i_{m}\right)} .
\end{aligned}
$$

Hence, $\mathcal{B}=P \mathcal{A} P^{T}$. Thus, $\mathcal{A}$ and $\mathcal{B}$ are permutational similar.

By Theorem 1 and Remark 3, we have the following expression theorem of permutational transformation of tensors.

Theorem 2 Let $\mathcal{A} \in \mathbb{C}^{[m, n]}$, and $\pi$ be a permutation on [ $\left.n\right]$. Then

$$
P_{\pi}(\mathcal{A})=P \mathcal{A} P^{T}
$$

where $P_{i j}=1 \Longleftrightarrow j=\pi(i) ; P_{i j}=0$, otherwise.

\section{Basic properties of permutation transformations}

Now, we discuss basic properties of permutation transformation of tensors. Firstly, we present some definitions and a lemma, which are needed in the subsequent analysis. For an $n$-dimensional vector $x=\left(x_{1}, x_{2}, \ldots, x_{n}\right)$, real or complex, we define the $n$-dimensional vector: 


$$
\mathcal{A} x^{m-1}:=\left(\sum_{i_{2}, \ldots, i_{m} \in[n]} a_{i i_{2} \ldots i_{m}} x_{i_{2}} \ldots x_{i_{m}}\right)_{1 \leq i \leq n}
$$

and the $n$-dimensional vector:

$$
x^{[m-1]}:=\left(x_{i}^{m-1}\right)_{1 \leq i \leq n} .
$$

The following two definitions were first introduced and studied by Lim (2005) and Qi (2005).

Definition 8 (Lim 2005; Qi 2005) Let $\mathcal{A} \in \mathbb{R}^{[m, n]}$. A pair $(\lambda, x) \in \mathbb{C} \times\left(\mathbb{C}^{n} \backslash\{0\}\right)$ is called an eigenvalue-eigenvector (or simply eigenpair) of $\mathcal{A}$ if they satisfy the equation

$$
\mathcal{A} x^{m-1}=\lambda x^{[m-1]} .
$$

We call $(\lambda, x)$ an $\mathrm{H}$-eigenpair if they are both real.

The set of all eigenvalues of $\mathcal{A}$ is denoted by $\sigma(\mathcal{A})$ and it is called the spectral of $\mathcal{A}$. Let $\rho(\mathcal{A})=\max \{|\lambda|: \lambda \in \sigma(\mathcal{A})\}$. It is called the spectral radius of $\mathcal{A}$.

Definition 9 (Lim 2005; Qi 2005) Let $\mathcal{A} \in \mathbb{R}^{[m, n]}$. A pair $(\lambda, x) \in \mathbb{C} \times\left(\mathbb{C}^{n} \backslash\{0\}\right)$ is called an E-eigenvalue and E-eigenvector (or simply E-eigenpair) of $\mathcal{A}$ if they satisfy the equation

$$
\left\{\begin{array}{l}
\mathcal{A} x^{m-1}=\lambda x \\
x^{T} x=1
\end{array}\right.
$$

We call $(\lambda, x)$ an Z-eigenpair if they are both real.

Definition 10 (Zhang et al. 2014) A tensor $\mathcal{A}=\left(a_{i_{1} \ldots i_{m}}\right) \in \mathbb{R}^{[m, n]}$ is called strictly diagonally dominant if

$$
\left|a_{i i \ldots i}\right|>\sum_{\substack{i_{2}, \ldots, i_{m} \in[n], \delta_{i i_{2} \ldots m_{m}=0}}}\left|a_{i i_{2} \ldots i_{m}}\right|, \quad \forall i \in[n] .
$$

Definition 11 (Bu et al. 2014) A tensor $\mathcal{A} \in \mathbb{C}^{n_{1} \times \cdots \times n_{k}}$ is said to have rank-one if there exist nonzero $a_{i} \in \mathbb{C}^{n_{i}}(i=1, \ldots, k)$ such that $\mathcal{A}=a_{1} \otimes a_{2} \otimes \cdots \otimes a_{k}$, where $a_{1} \otimes a_{2} \otimes \cdots \otimes a_{k}$ is the segre outer product of $a_{1} \in \mathbb{C}^{n_{1}}, \ldots, a_{k} \in \mathbb{C}^{n_{k}}$ with entries $a_{i_{1} \ldots i_{k}}=\left(a_{1}\right)_{i_{1}} \ldots\left(a_{k}\right)_{i_{k}}$. The rank of a tensor $\mathcal{A}$, denoted by $\operatorname{rank}(\mathcal{A})$, is defined to be the smallest $r$ such that $\mathcal{A}$ can be written as a sum of $r$ rank-one tensors. Especially, if $\mathcal{A}=0$, then $\operatorname{rank}(\mathcal{A})=0$.

Lemma 1 (Qi 2005) Assume that $\mathcal{A} \in \mathbb{R}^{[m, n]}$ is an even-order symmetric tensor. The following conclusions hold for $\mathcal{A}$,

(i) $\mathcal{A}$ always has H-eigenvalues. $\mathcal{A}$ is positive definite (positive semidefinite) if and only if all of its $H$-eigenvalues are positive (nonnegative).

(ii) $\mathcal{A}$ always has $Z$-eigenvalues. $\mathcal{A}$ is positive definite (positive semidefinite) if and only if all of its $Z$-eigenvalues are positive (nonnegative). 
Next, we present some basic properties of permutation transformation of tensor as follows.

Theorem 3 Let $\mathcal{A}, \mathcal{B} \in \mathbb{C}^{[m, n]}\left(\mathbb{R}^{[m, n]}\right)$, and $\pi$ be a permutation on $[n]$. Then

(i) $\quad P_{\pi}(\mathcal{A}+\mathcal{B})=P_{\pi}(\mathcal{A})+P_{\pi}(\mathcal{B})$.

(ii) $\quad P_{\pi}(k \mathcal{A})=k P_{\pi}(\mathcal{A})$, where $k \in \mathbb{C}(\mathbb{R})$.

(iii) $P_{\pi}(\mathcal{I})=\mathcal{I}$.

(iv) $P_{\pi}^{-1}\left(P_{\pi}(\mathcal{A})\right)=\mathcal{A}$.

(v) $P_{\pi}\left(P_{\pi}^{-1}(\mathcal{A})\right)=\mathcal{A}$.

(vi) $\sigma\left(P_{\pi}(\mathcal{A})\right)=\sigma(\mathcal{A})$.

(vii) $\rho\left(P_{\pi}(\mathcal{A})\right)=\rho(\mathcal{A})$.

(viii) $\operatorname{rank}\left(P_{\pi}(\mathcal{A})\right)=\operatorname{rank}(\mathcal{A})$.

(ix) If $\mathcal{A} \in \mathbb{S}^{[m, n]}$, then $P_{\pi}(\mathcal{A}) \in \mathbb{S}^{[m, n]}$.

(x) If $\mathcal{A}$ is a strictly diagonally dominant tensor, then $P_{\pi}(\mathcal{A})$ is also a strictly diagonally dominant tensor.

(xi) If $m$ is even and $\mathcal{A} \in \mathbb{S}^{[m, n]}$ is positive definite (positive semidefinite) tensor, then $P_{\pi}(\mathcal{A}) \in \mathbb{S}^{[m, n]}$ and is also a positive definite (positive semidefinite) tensor.

Proof (i) $P_{\pi}(\mathcal{A}+\mathcal{B})_{i_{1} \ldots i_{m}}=a_{\pi\left(i_{1}\right) \ldots \pi\left(i_{m}\right)}+b_{\pi\left(i_{1}\right) \ldots \pi\left(i_{m}\right)}=\left(P_{\pi}(\mathcal{A})+P_{\pi}(\mathcal{B})\right)_{i_{1} \ldots i_{m}}$.

(ii) $P_{\pi}(k \mathcal{A})_{i_{1} \ldots i_{m}}=k a_{\pi\left(i_{1}\right) \ldots \pi\left(i_{m}\right)}=k P_{\pi}(\mathcal{A})_{i_{1} \ldots i_{m}}$.

(iii) Since $P_{\pi}(\mathcal{I})_{i_{1} \ldots i_{m}}=\mathcal{I}_{\pi\left(i_{1}\right) \ldots \pi\left(i_{m}\right)}$ and $\delta_{\pi\left(i_{1}\right) \ldots \pi\left(i_{m}\right)}=1$ if and only if $\delta_{i_{1} \ldots i_{m}}=1$, then $P_{\pi}(\mathcal{I})=\mathcal{I}$.

From the Definition 3, it is easy to obtain (iv) and (v) are hold.

(vi) By Theorem $1, P_{\pi}(\mathcal{A})$ and $\mathcal{A}$ are permutational similar. By Theorem 2.1 in Shao (2013),

$\phi_{P_{\pi}(\mathcal{A})}(\lambda)=\phi_{\mathcal{A}}(\lambda)$,

where $\phi_{\mathcal{A}}(\lambda)$ is the characteristic polynomial of the tensor $\mathcal{A}$. In Qi (2005), Qi has proved that a number $\lambda \in \mathbb{C}$ is an eigenvalue of $\mathcal{A}$ if and only if it is a root of $\phi_{\mathcal{A}}(\lambda)$. Hence, similar tensors have the same eigenvalues. Then

$\sigma(\mathcal{A})=\sigma\left(P_{\pi}(\mathcal{A})\right)$

(vii) It is easy to be got from the results of (vi).

(viii) Let $\operatorname{rank}(\mathcal{A})=r$, and $P_{\pi}(\mathcal{A})=\left(a_{\pi\left(i_{1}\right) \ldots \pi\left(i_{m}\right)}\right)$, where $\pi$ is a permutation on $[n]$.

Case 1. If $r=1$, then there exists $a_{i} \in \mathbb{C}^{n_{i}}\left(i \in[m], a_{i} \neq 0\right)$ such that

$\mathcal{A}=a_{1} \otimes a_{2} \otimes \cdots \otimes a_{m}$

which implies,

$a_{i_{1} \ldots i_{m}}=\left(a_{1}\right)_{i_{1}}\left(a_{2}\right)_{i_{2}} \ldots\left(a_{m}\right)_{i_{m}}$.

Therefore,

$$
\begin{aligned}
a_{\pi\left(i_{1}\right) \pi\left(i_{2}\right) \ldots \pi\left(i_{m}\right)} & =\left(a_{1}\right)_{\pi\left(i_{1}\right)}\left(a_{2}\right)_{\pi\left(i_{2}\right)} \ldots\left(a_{m}\right)_{\pi\left(i_{m}\right)} \\
& =\left(P_{\pi}\left(a_{1}\right)\right)_{i_{1}}\left(P_{\pi}\left(a_{2}\right)\right)_{i_{2}} \ldots\left(P_{\pi}\left(a_{m}\right)\right)_{i_{m}} .
\end{aligned}
$$

Hence, $\quad P_{\pi}(\mathcal{A})=P_{\pi}\left(a_{1}\right) \otimes P_{\pi}\left(a_{2}\right) \otimes \cdots \otimes P_{\pi}\left(a_{m}\right)$. Since $\quad P_{\pi}\left(a_{i}\right) \in \mathbb{C}^{n_{i}} \quad$ and $P_{\pi}\left(a_{i}\right) \neq 0$, then $\operatorname{rank}\left(P_{\pi}(\mathcal{A})\right)=1$. 
Case 2. If $r>1$, then $\mathcal{A}$ can be written at least as a sum of $r$ rank-one tensors. Let $\mathcal{A}=\sum_{i \in[r]} \mathcal{A}_{i}$, where $\mathcal{A}_{i} \in \mathbb{C}^{[m, n]}, \operatorname{rank}\left(\mathcal{A}_{i}\right)=1, i \in[r]$. Then,

$P_{\pi}(\mathcal{A})=\sum_{i \in[r]} P_{\pi}\left(\mathcal{A}_{i}\right)$

By the results of Case 1, we have

$\operatorname{rank}\left(P_{\pi}\left(\mathcal{A}_{i}\right)\right)=1, i \in[r]$.

Hence, $\operatorname{rank}\left(P_{\pi}(\mathcal{A})\right) \leq r$. Next, we will prove that $\operatorname{rank}\left(P_{\pi}(\mathcal{A})\right)<r$ is impossible. Suppose that $\operatorname{rank}\left(P_{\pi}(\mathcal{A})\right)=r^{\prime}<r$. Then $P_{\pi}(\mathcal{A})$ can be written at least as a sum of $r^{\prime}$ rank-one tensors as follows

$P_{\pi}(\mathcal{A})=\sum_{i \in\left[r^{\prime}\right]} \mathcal{D}_{i}, \quad$ where $\quad \mathcal{D}_{i} \in \mathbb{C}^{[m, n]}, \quad \operatorname{rank}\left(\mathcal{D}_{i}\right)=1, i \in\left[r^{\prime}\right]$,

and $\pi^{-1}$ be the inverse transformation of $\pi$ on $[n]$. Then from (iv),

$\mathcal{A}=P_{\pi}^{-1}\left(P_{\pi}(\mathcal{A})\right)=\sum_{i \in\left[r^{\prime}\right]} P_{\pi^{-1}}\left(\mathcal{D}_{i}\right)$

From case $1, \operatorname{rank}\left(P_{\pi}^{-1}\left(\mathcal{D}_{i}\right)\right)=1$, so $\operatorname{rank}(\mathcal{A}) \leq r^{\prime}<r$. It's a contradiction. Therefore, $\operatorname{rank}\left(P_{\pi}(\mathcal{A})\right)=r$.

(ix) It is easy to be proved from the definition of symmetric tensors.

(x) Suppose that $\mathcal{A}$ is a strictly diagonally dominant tensor, then

$$
\begin{aligned}
\left|P_{\pi}(\mathcal{A})_{i i \ldots i}\right| & =\left|\mathcal{A}_{\pi(i) \pi(i) \ldots \pi(i)}\right| \\
& >\sum_{\substack{i_{2}, \ldots, i_{m} \in[n], \delta_{\pi(i) i_{2} \ldots i i_{m}=0}}}\left|a_{\pi(i) i_{2} \ldots i_{m}}\right| \\
& =\sum_{\substack{i_{2}, \ldots, i_{m} \in[n], \delta_{i i_{2} \ldots i_{m}=0}}}\left|P_{\pi}(\mathcal{A})_{i i_{2} \ldots i_{m}}\right|, \forall i \in[n] .
\end{aligned}
$$

Thus, $P_{\pi}(\mathcal{A})$ is also a strictly diagonally dominant tensor.

(xi) Suppose that $\mathcal{A}$ is positive definite (semidefinite), then all $\mathrm{H}$-eigenvalues of $\mathcal{A}$ are positive (nonnegative). By (vi) and (ix) of Theorem 3, $P_{\pi}(\mathcal{A})$ is an even-order symmetric tensor, and $\sigma\left(P_{\pi}(\mathcal{A})\right)=\sigma(\mathcal{A})$. From the results above, we know that all $\mathrm{H}$-eigenvalues of $P_{\pi}(\mathcal{A})$ are positive (nonnegative). Then, by Lemma $1, P_{\pi}(\mathcal{A})$ is positive definite (positive semidefinite).

Remark 4 By the results of (i) and (ii) of Theorem 3, we know that a permutation transformation of tensor is a linear transformation on $\mathbb{C}^{[m, n]}\left(\mathbb{R}^{[m, n]}\right)$.

\section{Permutation transformation on some structure tensors}

It is universally acknowledged that some structure tensors with good properties have been well studied, such as nonnegative tensor, symmetric tensor, positive definite (positive semidefinite) tensor, $Z$-tensor, (strong) $M$-tensor, Hankel tensor, $P\left(P_{0}\right)$-tensor, $B\left(B_{0}\right)$-tensor and $H$-tensor. In this section, we discuss the invariance under permutation transformations for some important structure tensors. 
Definition 12 (Chang et al. 2008) A tensor $\mathcal{A}=\left(a_{i_{1} \ldots i_{m}}\right) \in \mathbb{R}^{[m, n]}$ is called a nonnegative tensor, denoted by $\mathcal{A} \geq 0$, if each entry is nonnegative.

Definition 13 (Zhang et al. 2014) We call a tensor $\mathcal{A}$ as an $Z$-tensor, if all of its offdiagonal entries are non-positive, which is equivalent to write $\mathcal{A}=s \mathcal{I}-\mathcal{B}$, where $s>0$ and $\mathcal{B}$ is a nonnegative tensor.

From Definition 12, we easily get the following two lemmas.

Lemma 2 Let $\mathcal{A} \in \mathbb{R}^{[m, n]}$ be a nonnegative tensor, $\pi$ be a permutation on [ $\left.n\right]$. Then $P_{\pi}(\mathcal{A})$ is also a nonnegative tensor.

Lemma 3 Let $\mathcal{A} \in \mathbb{R}^{[m, n]}$ be an Z-tensor, and $\pi$ be a permutation on $[n]$. Then $P_{\pi}(\mathcal{A})$ is also an Z-tensor.

Definition 14 (Zhang et al. 2014) We call an $Z$-tensor $\mathcal{A}=s \mathcal{I}-\mathcal{B}(\mathcal{B} \geq 0)$ as an $M$-tensor if $s \geq \rho(\mathcal{B})$; we call it as a strong $M$-tensor if $s>\rho(\mathcal{B})$, where $\rho(\mathcal{B})$ is the spectral radius of $\mathcal{B}$.

Theorem 4 Let $\mathcal{A} \in \mathbb{R}^{[m, n]}$ be an (strong) M-tensor, and $\pi$ be a permutation on [ $\left.n\right]$. Then $P_{\pi}(\mathcal{A})$ is also an (strong) M-tensor.

Proof Suppose that $\mathcal{A} \in \mathbb{R}^{[m, n]}$ is an (strong) $M$-tensor, then there exist a nonnegative tensor $\mathcal{B}$ and a real number $s \geq \rho(\mathcal{B})(s>\rho(\mathcal{B}))$ such that

$$
\mathcal{A}=s \mathcal{I}-\mathcal{B} .
$$

From Remark 3,

$$
P_{\pi}(\mathcal{A})=s \mathcal{I}-P_{\pi}(\mathcal{B})
$$

By Lemma $3, P_{\pi}(\mathcal{A})$ is an $Z$-tensor. It follows from (vii) of Theorem 3 that $\rho\left(P_{\pi}(\mathcal{B})\right)=\rho(\mathcal{B})$, which implies

$$
s \geq \rho\left(P_{\pi}(\mathcal{B})\right)\left(s>\rho\left(P_{\pi}(\mathcal{B})\right)\right) .
$$

Therefore, $P_{\pi}(\mathcal{A})$ is an (strong) $M$-tensor.

Definition 15 (Song and Qi 2014) Let $\mathcal{A}=\left(a_{i_{1} i_{2} \ldots i_{m}}\right) \in \mathbb{R}^{[m, n]}$. We say that $\mathcal{A}$ is

(i) a $P_{0}$-tensor if for any nonzero vector $x \in \mathbb{R}^{n}$, there exists $i \in[n]$ such that $x_{i} \neq 0$ and $x_{i}\left(\mathcal{A} x^{m-1}\right)_{i} \geq 0$

(ii) a $P$-tensor if for any nonzero vector $x \in \mathbb{R}^{n}, \max _{i \in[n]} x_{i}\left(\mathcal{A} x^{m-1}\right)_{i}>0$.

Lemma 4 (Song and Qi 2014) A symmetric tensor is a $P\left(P_{0}\right)$-tensor if and only if it is positive (semi)definite.

Theorem 5 Let $\mathcal{A} \in \mathbb{S}^{[m, n]}$ be an even-order $P\left(P_{0}\right)$-tensor, and $\pi$ be a permutation on $[n]$. Then $P_{\pi}(\mathcal{A})$ is also an even-order symmetric $P\left(P_{0}\right)$-tensor. 
Proof Suppose that $\mathcal{A}$ is an even-order symmetric $P\left(P_{0}\right)$-tensor. According to Lemma $4, \mathcal{A}$ is positive (semi)definite. It follows from (ix) of Theorem 3 that $P_{\pi}(\mathcal{A})$ is positive (semi)definite. Hence, by Lemma $4, P_{\pi}(\mathcal{A})$ is a $P\left(P_{0}\right)$-tensor.

Definition 16 (Song and Qi 2014) Let $\mathcal{A}=\left(a_{i_{1} i_{2} \ldots i_{m}}\right) \in \mathbb{R}^{[m, n]}$. We say that $\mathcal{A}$ is a $B$ -tensor, if for all $i \in[n]$,

$$
\sum_{i_{2}, \ldots, i_{m} \in[n]} a_{i i_{2} \ldots i_{m}}>0,
$$

and

$$
\frac{1}{n^{m-1}}\left(\sum_{i_{2}, \ldots, i_{m} \in[n]} a_{i i_{2} \ldots i_{m}}\right)>a_{i j_{2} \ldots j_{m}},
$$

for all $\left(j_{2}, \ldots, j_{m}\right) \neq(i, \ldots, i)$. We say that $\mathcal{A}$ is a $B_{0}$-tensor, if for all $i \in[n]$,

$$
\sum_{i_{2}, \ldots, i_{m} \in[n]} a_{i i_{2} \ldots i_{m}} \geq 0,
$$

and

$$
\frac{1}{n^{m-1}}\left(\sum_{i_{2}, \ldots, i_{m} \in[n]} a_{i i_{2} \ldots i_{m}}\right) \geq a_{i j_{2} \ldots j_{m}}
$$

for all $\left(j_{2}, \ldots, j_{m}\right) \neq(i, \ldots, i)$.

Theorem 6 Let $\mathcal{A} \in \mathbb{R}^{[m, n]}$ be a $B\left(B_{0}\right)$-tensor, and $\pi$ be a permutation on [ $\left.n\right]$. Then $P_{\pi}(\mathcal{A})$ is also a $B\left(B_{0}\right)$-tensor.

Proof Suppose that $\mathcal{A}=\left(a_{i_{1} i_{2} \ldots i_{m}}\right)$ is a $B$-tensor, then for all $i \in[n]$,

$$
\sum_{i_{2}, \ldots, i_{m} \in[n]} a_{i i_{2} \ldots i_{m}}>0
$$

and

$$
\frac{1}{n^{m-1}}\left(\sum_{i_{2}, \ldots, i_{m} \in[n]} a_{i i_{2} \ldots i_{m}}\right)>a_{i j_{2} \ldots j_{m}},
$$

for all $\left(j_{2}, \ldots, j_{m}\right) \neq(i, \ldots, i)$.

$$
\text { Let } P_{\pi}(\mathcal{A})=\left(b_{i_{1} i_{2} \ldots i_{m}}\right) \text {. Then }
$$

$$
\begin{aligned}
\sum_{i_{2}, \ldots, i_{m} \in[n]} b_{i i_{2} \ldots i_{m}} & =\sum_{i_{2}, \ldots, i_{m} \in[n]} a_{\pi(i) \pi\left(i_{2}\right) \ldots \pi\left(i_{m}\right)} \\
& =\sum_{\pi\left(i_{2}\right), \ldots, \pi\left(i_{m}\right) \in[n]} a_{\pi(i) \pi\left(i_{2}\right) \ldots \pi\left(i_{m}\right)}>0,
\end{aligned}
$$


and

$$
\begin{aligned}
\frac{1}{n^{m-1}}\left(\sum_{i_{2}, \ldots, i_{m} \in[n]} b_{i i_{2} \ldots i_{m}}\right) & =\frac{1}{n^{m-1}}\left(\sum_{i_{2}, \ldots, i_{m} \in[n]} a_{\pi(i) \pi\left(i_{2}\right) \ldots \pi\left(i_{m}\right)}\right) \\
& =\frac{1}{n^{m-1}}\left(\sum_{\pi\left(i_{2}\right), \ldots, \pi\left(i_{m}\right) \in[n]} a_{\pi(i) \pi\left(i_{2}\right) \ldots \pi\left(i_{m}\right)}\right) \\
& >a_{\pi(i) \pi\left(j_{2}\right) \ldots \pi\left(j_{m}\right)}=b_{i j_{2} \ldots j_{m} .}
\end{aligned}
$$

Hence, $P_{\pi}(\mathcal{A})$ is a $B$-tensor. Similarly, we can prove that the case of $B_{0}$-tensor.

Definition 17 (Li et al. 2014) A tensor $\mathcal{A}=\left(a_{i_{1} i_{2} \ldots i_{m}}\right) \in \mathbb{C}^{[m, n]}$ is called an $H$-tensor if there is an entrywise positive vector $x=\left(x_{1}, x_{2}, \ldots, x_{n}\right)^{T} \in \mathbb{R}^{n}$ such that for all $i \in[n]$,

$$
\left|a_{i \ldots i}\right| x_{i}^{m-1}>\sum_{\substack{i_{2}, \ldots, i_{m} \in[n] \\ \delta_{i i_{2} \ldots i_{m}}=0}}\left|a_{i i_{2} \ldots i_{m}}\right| x_{i_{2}} \ldots x_{i_{m}}
$$

Theorem 7 Let $\mathcal{A} \in \mathbb{C}^{[m, n]}$ be an $H$-tensor, and $\pi$ be a permutation on $[n]$. Then $P_{\pi}(\mathcal{A})$ is also an $\mathrm{H}$-tensor.

Proof Suppose that $\mathcal{A}=\left(a_{i_{1} i_{2} \ldots i_{m}}\right)$ is an $H$-tensor. Then there is an entrywise positive vector $x=\left(x_{1}, x_{2}, \ldots, x_{n}\right)^{T} \in \mathbb{R}^{n}$ such that for all $i \in[n]$, the Inequality 3 holds. Let $P_{\pi}(\mathcal{A})=\left(b_{i_{1} i_{2} \ldots i_{m}}\right)$, where $b_{i_{1} i_{2} \ldots i_{m}}=a_{\pi\left(i_{1}\right) \pi\left(i_{2}\right) \ldots \pi\left(i_{m}\right)}$. Hence, for all $i \in[n]$,

$$
\begin{aligned}
\left|b_{i \ldots i}\right| x_{i}^{m-1} & =\left|a_{\pi(i) \ldots \pi(i)}\right| x_{i}^{m-1} \\
& >\sum_{\substack{i_{2}, \ldots, i_{m} \in[n], \delta_{\pi(i) i_{2} \ldots i_{m}}=0}}\left|a_{\pi(i) i_{2} \ldots i_{m}}\right| x_{i_{2}} \ldots x_{i_{m}} \\
& =\sum_{\substack{i_{2}, \ldots, i_{m} \in[n], \delta_{i i_{2} \ldots i_{m}}=0}}\left|b_{i i_{2} \ldots i_{m}}\right| x_{i_{2}} \ldots x_{i_{m}} \\
& =\sum_{\substack{i_{2}, \ldots, i_{m} \in[n] \\
\delta_{i i_{2} \ldots i_{m}}=0}}\left|b_{i i_{2} \ldots i_{m}}\right| x_{i_{2}} \ldots x_{i_{m}} .
\end{aligned}
$$

Thus, $P_{\pi}(\mathcal{A})$ is an $H$-tensor.

\section{Canonical form of tensors}

In this section, as an application of the permutation transformation of tensors, we would introduce some results which show that the canonical form theorem for matrices could be generalized to tensors. For the convenience discussion, we starts with the following definitions and lemmas.

Let $\mathcal{A} \in \mathbb{R}^{[m, n]}$ be a nonnegative tensor. The directed graph $G(\mathcal{A})$ (Chang et al. 2013; Friedland et al. 2013) associated to $\mathcal{A}$ is the directed graph with vertices $1,2, \ldots, n$ and an edge from $i$ to $j$ if and only if $a_{i i_{2} \ldots i_{m}} \neq 0$ for some $i_{l}=j, l=2,3, \ldots, m$. 
Definition 18 (Chang et al. 2013; Friedland et al. 2013; Hu et al. 2014) A nonnegative tensor $\mathcal{A} \in \mathbb{R}^{[m, n]}$ is called weakly irreducible if the associate directed graph $G(\mathcal{A})$ is strongly connected. A tensor $\mathcal{A}$ is said to be weakly irreducible if $|\mathcal{A}|$ is weakly irreducible, where $|\mathcal{A}|$ denote the tensor whose $\left(i_{1}, \ldots, i_{m}\right)$-th entry is defined as $\left|a_{i_{1} \ldots i_{m}}\right|$.

Definition 19 (Shao et al. 2013) Let $\mathcal{A}=\left(a_{i_{1} \ldots i_{m}}\right) \in \mathbb{R}^{[m, n]}$. If there exists some integer $k$ with $1 \leq k \leq n-1$ such that

$$
a_{i_{1} i_{2} \ldots i_{m}}=0 \text {, }
$$

for all $i_{1} \leq k$ and at least one of $\left\{i_{2}, \ldots, i_{m}\right\}$ is greater than $k$, then $\mathcal{A}$ is called a $k$-lower triangular block tensor, or simply a lower triangular block tensor.

Definition 20 Let $\mathcal{A} \in \mathbb{R}^{[m, n]}$ and $\alpha \subseteq[n]$. A principal subtensor $\mathcal{A}[\alpha]$ of the tensor $\mathcal{A}$ is defined as an order $m$ dimensional $|\alpha|$ tensor with entries

$$
\mathcal{A}[\alpha]=\left(a_{i_{1} \ldots i_{m}}\right), \quad i_{1}, \ldots, i_{m} \in \alpha .
$$

Definition 21 (Shao et al. 2013) Let $\mathcal{A} \in \mathbb{R}^{[m, n]}$, and $n_{1}, \ldots, n_{r}$ be positive integers with $n_{1}+\cdots+n_{r}=n(r \geq 1)$. Let

$$
\begin{aligned}
I_{1} & =\left\{1,2, \ldots, n_{1}\right\}, \\
I_{i} & =\left\{\left(\sum_{j \in[i-1]} n_{j}\right)+1, \ldots, \sum_{j \in[i]} n_{j}\right\} \subseteq[n](i \in[r] \backslash\{1\}),
\end{aligned}
$$

and write $\mathcal{A}\left[I_{i}\right]=\mathcal{A}_{i}$. Suppose that for each $1 \leq i \leq r-1$, the subtensor $\mathcal{A}\left[I_{i} \cup \cdots \cup I_{r}\right]$ is a $n_{i}$-lower triangular block tensor, then $\mathcal{A}$ is called a $\left(n_{1}, \ldots, n_{r}\right)$-lower triangular block tensor with the diagonal blocks $\mathcal{A}_{1}, \ldots, \mathcal{A}_{r}$.

Lemma 5 (Shao et al. 2013) Let $\mathcal{A} \in \mathbb{R}^{[m, n]}$ and $m \geq 2$. Then there exists positive integers $r \geq 1$ and $n_{1}, \ldots, n_{r}$ with $n_{1}+\cdots+n_{r}=n(r \geq 1)$ such that $\mathcal{A}$ is permutational similar to some $\left(n_{1}+\cdots+n_{r}\right)$-lower triangular block tensor, where all the diagonal blocks $\mathcal{A}_{1}, \ldots, \mathcal{A}_{r}$ are weakly irreducible.

Lemma 6 (Shao et al. 2013) Let $r \geq 2$ and $n_{1}, \ldots, n_{r}$ be positive integers with $n_{1}+\cdots+n_{r}=n$. Let $\mathcal{A}$ be $a\left(n_{1}+\cdots+n_{r}\right)$-lower triangular block tensor with the diagonal blocks $\mathcal{A}_{1}, \ldots, \mathcal{A}_{r}$. Then we have:

$$
\operatorname{Det}(\mathcal{A})=\prod_{i \in[r]}\left(\operatorname{Det} \mathcal{A}_{i}\right)^{(m-1)^{n-n_{i}}}
$$

and thus

$$
\phi_{\mathcal{A}}(\lambda)=\prod_{i \in[r]}\left(\phi_{\mathcal{A}_{i}}(\lambda)\right)^{(m-1)^{n-n_{i}}}
$$

where $\phi_{\mathcal{A}}(\lambda)$ is the characteristic polynomial of the tensor $\mathcal{A}$. 
From Theorem 1, Lemmas 5 and 6, we can easily obtain the following theorems.

Theorem 8 Let $\mathcal{A} \in \mathbb{R}^{[m, n]}$, then there exists a permutation $\pi$ on $[n]$ such that $P_{\pi}(\mathcal{A})$ is $a\left(n_{1}+\cdots+n_{r}\right)$-lower triangular block tensor, where all the diagonal blocks $\mathcal{A}_{1}, \ldots, \mathcal{A}_{r}$ are weakly irreducible.

Remark 5 Theorem 8 could be called the "canonical form" theorem and the $\left(n_{1}+\cdots+n_{r}\right)$-lower triangular block tensor is called the "canonical form" of $\mathcal{A}$.

Theorem 9 Let $\mathcal{A} \in \mathbb{R}^{[m, n]}$ and $\overline{\mathcal{A}}$ be the "canonical form" of $\mathcal{A}$. Then

$$
\sigma(\mathcal{A})=\sigma(\overline{\mathcal{A}})=\bigcup_{i \in[r]} \sigma\left(\mathcal{A}_{i}\right)
$$

Proof From Theorem 8 and (vi) of Theorem 3, we have

$$
\sigma(\mathcal{A})=\sigma(\overline{\mathcal{A}})
$$

From Lemma 6, we have

$$
\sigma(\overline{\mathcal{A}})=\bigcup_{i \in[r]} \sigma\left(\mathcal{A}_{i}\right)
$$

Therefore, the conclusion holds.

Canonical form of tensors plays an important role in decomposition of symmetric tensors as a sum of component rank-one tensors, which has numerous applications in electrical engineering and higher order statistics (Brachat et al. 2009; Kolda and Bader 2009; Robeva 2016), such Independent Component Analysis (Comon 1992). Given a symmetric tensor $\mathcal{A} \in \mathbb{R}^{[m, n]}$, the aim is to decompose it as

$$
\mathcal{A}=\sum_{i \in[k]} \lambda_{i} v_{i}^{\otimes m}
$$

where $v_{1}, \ldots, v_{k} \in \mathbb{R}^{n}$ and $\lambda_{1}, \ldots, \lambda_{k} \in \mathbb{R}$. Comon et al. (2008) showed that the decomposition of the form (4) exists. Furthermore, in Brachat et al. (2009) an algorithm for decomposition a symmetric tensor into a sum of component rank-one tensors was presented. However, for a tensor with large order or dimension the algorithm may result in large computing quantity. According to Theorem 8 , and taking into account that $\mathcal{A}$ is symmetric, there exists a permutation $\pi$ on $[n]$ such that $P_{\pi}(\mathcal{A})$ is a diagonal block tensor, where all the diagonal blocks $\mathcal{A}_{1} \in \mathbb{R}^{\left[m, n_{1}\right]}, \ldots, \mathcal{A}_{r} \in \mathbb{R}^{\left[m, n_{r}\right]}\left(\sum_{i \in[r]} n_{i}=n\right)$ are weakly irreducible. Therefore, we can use algorithm in Brachat et al. (2009) decomposition of lower dimensional symmetric tensor $\mathcal{A}_{j}, j \in[r]$ as follows

$$
\mathcal{A}_{j}=\sum_{i \in\left[k_{j}\right]} \lambda_{j i} v_{j i}^{\otimes m}, \quad j \in[r], v_{j i} \in \mathbb{R}^{n_{j}} .
$$


Therefore,

$$
P_{\pi}(\mathcal{A})=\sum_{j \in[r]} \sum_{i \in\left[k_{j}\right]} \lambda_{j i} \omega_{j i}^{\otimes m}
$$

where

$$
\omega_{j i}=(\overbrace{0, \ldots, 0}^{n_{1} \text { times }}, \ldots \overbrace{0, \ldots, 0}^{n_{j-1} \text { times }},\left(v_{j i}\right)^{T}, \overbrace{0, \ldots, 0}^{n_{j+1} \text { times }}, \ldots, \overbrace{0, \ldots, 0}^{n_{r} \text { times }})^{T} \in \mathbb{R}^{n}, j \in[r], i \in\left[k_{j}\right],
$$

thus, we obtain decomposition of the tensor $\mathcal{A}$ as follows.

$$
\mathcal{A}=\sum_{j \in[r]} \sum_{i \in\left[k_{j}\right]} \lambda_{j i}\left(P_{\pi}^{-1}\left(\omega_{j i}\right)\right)^{\otimes m} .
$$

We shall employ an example to illustrate this interesting property.

Example 1 Let $\mathcal{A}=\left(a_{i_{1} i_{2} i_{3} i_{4}}\right) \in \mathbb{R}^{[4,4]}$ be a symmetric tensor defined by

$$
\begin{aligned}
& a_{1111}=3, a_{2222}=15, a_{3333}=18, a_{1333}=a_{3133}=a_{3313}=a_{3331}=6, \\
& a_{1133}=a_{1313}=a_{1331}=a_{3113}=a_{3131}=a_{3311}=6, \\
& a_{2224}=a_{2242}=a_{2422}=a_{4222}=9, a_{2444}=a_{4244}=a_{4424}=a_{4442}=3, \\
& a_{2244}=a_{2424}=a_{2442}=a_{4224}=a_{4242}=a_{4422}=3, \text { other, } a_{i_{1} i_{2} i_{3} i_{4}}=0 .
\end{aligned}
$$

By Theorem 8 , we can obtain the canonical form of $\mathcal{A}$ as follows. Let $\pi$ be a permutation on $\{1,2,3,4\}$, where $\pi(1)=1, \pi(2)=3, \pi(3)=2, \pi(4)=4$. Therefore, $P_{\pi}(\mathcal{A})=\left(b_{i_{1} i_{2} i_{3} i_{4}}\right) \in \mathbb{R}^{[4,4]}$ reads as follows

$$
\begin{aligned}
& b_{1111}=3, b_{2222}=18, b_{3333}=15, b_{1222}=b_{2122}=b_{2212}=b_{2221}=6, \\
& b_{1122}=b_{1212}=b_{1221}=b_{2112}=b_{2121}=b_{2211}=6, \\
& b_{3334}=b_{3343}=b_{3433}=b_{4333}=9, b_{3444}=b_{4344}=b_{4434}=b_{4443}=3, \\
& b_{3344}=b_{3434}=b_{3443}=b_{4334}=b_{4343}=b_{4433}=3, \text { other, } b_{i_{1} i_{2} i_{3} i_{4}}=0 .
\end{aligned}
$$

It is easy to obtain that $P_{\pi}(\mathcal{A})$ is a diagonal block tensor with the diagonal blocks $P_{\pi}(\mathcal{A})\left[I_{j}\right], j \in\{1,2\}$, where $I_{1}=\{1,2\}, I_{2}=\{3,4\}$. By algorithm 1 in Brachat et al. (2009), we obtain the rank-one decomposition of the diagonal block tensors $P_{\pi}(\mathcal{A})\left[I_{j}\right], j \in\{1,2\}$ as follows

$$
\begin{aligned}
& P_{\pi}(\mathcal{A})\left[I_{1}\right]=v_{11}^{\otimes 4}+2 v_{12}^{\otimes 4}, \\
& P_{\pi}(\mathcal{A})\left[I_{2}\right]=v_{21}^{\otimes 4}-v_{22}^{\otimes 4},
\end{aligned}
$$

where

$$
v_{11}=(1,2)^{T}, \quad v_{12}=(1,-1)^{T}, \quad v_{21}=(2,1)^{T}, \quad v_{22}=(1,-1)^{T} .
$$

Therefore

$$
P_{\pi}(\mathcal{A})=\omega_{11}^{\otimes 4}+2 \omega_{12}^{\otimes 4}+\omega_{21}^{\otimes 4}-\omega_{22}^{\otimes 4}
$$


where

$$
\begin{array}{ll}
\omega_{11}=\left(v_{11}^{T}, 0,0\right)^{T}=(1,2,0,0)^{T}, & \omega_{12}=\left(v_{12}^{T}, 0,0\right)^{T}=(1,-1,0,0)^{T}, \\
\omega_{21}=\left(0,0, v_{21}^{T}\right)^{T}=(0,0,2,1)^{T}, & \omega_{22}=\left(0,0, v_{22}^{T}\right)^{T}=(0,0,1,-1)^{T} .
\end{array}
$$

which implies the rank-one decomposition of tensor $\mathcal{A}$ given by

$$
\mathcal{A}=\left(P_{\pi}^{-1}\left(\omega_{11}\right)\right)^{\otimes 4}+2\left(P_{\pi}^{-1}\left(\omega_{12}\right)\right)^{\otimes 4}+\left(P_{\pi}^{-1}\left(\omega_{21}\right)\right)^{\otimes 4}-\left(P_{\pi}^{-1}\left(\omega_{22}\right)\right)^{\otimes 4},
$$

where,

$$
\begin{array}{ll}
P_{\pi}^{-1}\left(\omega_{11}\right)=(1,0,2,0)^{T}, & P_{\pi}^{-1}\left(\omega_{12}\right)=(1,0,-1,0)^{T}, \\
P_{\pi}^{-1}\left(\omega_{21}\right)=(0,2,0,1)^{T}, & P_{\pi}^{-1}\left(\omega_{22}\right)=(0,1,0,-1)^{T} .
\end{array}
$$

\section{Conclusions}

In this paper, the definition of permutation transformation of tensors is introduced, and its properties are studied. As applications of permutation transformations, we give the canonical form theorem of tensors and a numerical example which show that some problems of higher dimension tensors can be translated into the corresponding problems of lower dimension weakly irreducible tensors by using the permutation transformation. There are many problems unsolved for permutation transformations of tensors and their applications. Hence, this paper is only a starting point for studying permutation transformations of tensors.

\section{Authors' contributions}

YTL, ZBL, QLL and QL are contributed equally to this work. All authors read and approved the final manuscript.

\section{Acknowledgements}

The authors are grateful to the anonymous referees for their valuable comments, which helped improve the quality of the paper. This work was supported by the National Nature Science Foundation of China (Grant No. 11361074).

\section{Competing interests}

The authors declare that they have no competing interests.

Received: 12 April 2016 Accepted: 21 November 2016

Published online: 28 November 2016

\section{References}

Brachat J, Comon P, Mourrain B, Tsigaridas E (2009) Symmetric tensor decomposition. In: Signal processing conference, 2009 17th European, pp 525-529

Bu C, Zhang X, Zhou J, Wang W, Wei Y (2014) The inverse, rank and product of tensors. Linear Algebra Appl 446:269-280

Chang K, Qi L, Zhang T (2013) A survey on the spectral theory of nonnegative tensors. Numer Linear Algebra Appl 20(6):891-912

Chang KC, Pearson K, Zhang T et al (2008) Perron-frobenius theorem for nonnegative tensors. Commun Math Sci 6(2):507-520

Comon P (1992) Independent component analysis. In: Lacoume J-L (ed) Higher-order statistics. Elsevier, Amsterdam, pp 29-38

Comon P, Golub G, Lim LH, Mourrain B (2008) Symmetric tensors and symmetric tensor rank. SIAM J Matrix Anal Appl 30(3):1254-1279

De Lathauwer L, De Moor B, Vandewalle J (2000) On the best rank-1 and rank- $\left(r_{1}, r_{2}, \ldots, r_{n}\right)$ approximation of higher-order tensors. SIAM J Matrix Anal Appl 21(4):1324-1342

Friedland S, Gaubert S, Han L (2013) Perron-frobenius theorem for nonnegative multilinear forms and extensions. Linear Algebra Appl 438(2):738-749

Hu S, Huang Z, Qi L (2014) Strictly nonnegative tensors and nonnegative tensor partition. Sci China Math 57(1):181-195

Kolda TG, Bader BW (2009) Tensor decompositions and applications. SIAM Rev 51(3):455-500

Li C, Wang F, Zhao J, Zhu Y, Li Y (2014) Criterions for the positive definiteness of real supersymmetric tensors. J Comput Appl Math 255:1-14 
Lim LH (2005) Singular values and eigenvalues of tensors: a variational approach. In: CAMSAP'05: proceeding of the IEEE international workshop on computational advances in multisensor adaptive pp 129-132

Liu Y, Zhou G, Ibrahim NF (2010) An always convergent algorithm for the largest eigenvalue of an irreducible nonnegative tensor. J Comput Appl Math 235(1):286-292

Ng M, Qi L, Zhou G (2009) Finding the largest eigenvalue of a nonnegative tensor. SIAM J Matrix Anal Appl 31(3):1090-1099

Qi L (2005) Eigenvalues of a real supersymmetric tensor. J Symb Comput 40(6):1302-1324

Robeva E (2016) Orthogonal decomposition of symmetric tensors. SIAM J Matrix Anal Appl 37(1):86-102

Shao JY (2013) A general product of tensors with applications. Linear Algebra Appl 439(8):2350-2366

Shao JY, Shan HY, Zhang L (2013) On some properties of the determinants of tensors. Linear Algebra Appl 439(10):3057-3069

Song Y, Qi L (2014) An initial study on p, p0, b and b0 tensors. arXiv preprint arXiv:14031118

Yang Y, Yang Q (2010) Further results for Perron-Frobenius theorem for nonnegative tensors. SIAM J Matrix Anal Appl 31(5):2517-2530

Zhang L, Qi L, Zhou G (2014) M-tensors and some applications. SIAM J Matrix Anal Appl 35(2):437-452

Zhang T, Golub GH (2001) Rank-one approximation to high order tensors. SIAM J Matrix Anal Appl 23(2):534-550

\section{Submit your manuscript to a SpringerOpen ${ }^{\circ}$ journal and benefit from:}

- Convenient online submission

\section{- Rigorous peer review}

- Immediate publication on acceptance

- Open access: articles freely available online

- High visibility within the field

- Retaining the copyright to your article 